\title{
Dose Optimization of Antibiotics in Renally Impaired Patients in Indian Settings: A Prospective Observational Study
}

\author{
Surabhi Sridhara ${ }^{1}$, Elstin Raj ${ }^{1}$, Prashant Chandra ${ }^{1}$, Ravindra A $^{2}$, Surulivelrajan \\ Mallayasamy ${ }^{1}$, and Rajesh $\mathrm{V}^{1}$ \\ ${ }^{1}$ Manipal College of Pharmaceutical Sciences \\ ${ }^{2}$ KMC Manipal
}

June 22, 2020

\begin{abstract}
Rationale: In renal impairment, the pharmacokinetic properties of the drug are altered, and the systemic clearance is reduced. The current study aimed to assess the optimization of antibiotics dosing in renally impaired patients in a healthcare setting. Methodology: A prospective study was conducted on in-patients in the nephrology department, prescribed with antibiotics in a tertiary care hospital. The creatinine clearance was calculated by Cockroft-Gault and Jelliffe method. The dose appropriateness was cross-checked using standard databases and literature from the manufacturer data. Results: Of 139 participants 112 (80.6\%) had CKD and 27(19.4\%) had AKI. Urinary tract infection was most common. Monotherapy (62) was the most preferred choice, followed by dual in (43) and triple in (7\%). A positive clinical outcome of $79.1 \%$ was achieved. Cefoperazone-sulbactam was most widely used antibiotic. The mean difference in creatinine clearance was $4.55 \mathrm{ml} / \mathrm{min}$ in AKI patients. Conclusion: Dose appropriateness is a significant factor in achieving favorable clinical outcomes.
\end{abstract}

\section{INTRODUCTION}

Appropriate antibiotic dosing is fundamental to achieve favourable clinical outcomes. Optimum dosing ensures adequate therapeutic drug concentrations at the site of infection. Acute Kidney Injury (AKI) and Chronic Kidney Disease (CKD) substantially alter drug pharmacokinetics. ${ }^{1}$ High mortality has been reported in hospitalized patients with AKI. Diverse etiology makes treatment challenging ${ }^{2}$. Quality of life in CKD decreases with declining GFR ${ }^{3}$.

Antibiotics are the most common drugs that require dose optimization but are often dosed inappropriately in patients with renal impairment ${ }^{4}$. Appropriate dosing schedules at right intervals helps in reducing morbidity, mortality, and length of hospital stay ${ }^{5}$. An inappropriate dose adjustment could result in elevated plasma concentrations of the drug leading to adverse drug reactions, thus increasing the patient's burden ${ }^{6}$. Antibiotic dose optimization is an essential part of clinical practice for better clinical management and delayed resistance $^{7}$. Antibiotic resistance not only increases healthcare costs but also worsens severity of infection ${ }^{8}$. Creatinine clearance $(\mathrm{CrCl})$ and estimated glomerular filtration rate (eGFR) are universally used clinical parameters in renally impaired patients. Variations in drug clearance are proportional to the changes in $\mathrm{CrCl}$. This association can serve as a basis for modifying the dosing interval of drugs that undergo renal elimination.

Regardless of the published guidelines, there is insufficient evidence on dosing decisions on many commonly used drugs for patients with deranged kidney function ${ }^{9}$. Definitive conclusions on the pharmacokinetic parameters of metabolized medications in AKI remains unanswered. Physicians and pharmacists commonly use Cockroft-Gaults formula (also recommended by USFDA) for estimating $\mathrm{CrCl}^{10,11}$. However, in AKI, a steady state kidney function is not maintained. Therefore, the Jelliffe method was developed to ascertain 
GFR in settings of non-steady-state kidney function. Calculation using the Jelliffe equation, modified by consideration of patient volume status, yielded a more consistent and precise valuation of kidney function when equated with planned collection of urine in AKI patients ${ }^{12}$. The Acute Disease Quality Initiative (ADQI) recommends the use of short timed urine collections or the modified Jelliffe equation for estimating kidney function in persistent $\mathrm{AKI}^{13}$.

The current study aimed to assess the different aspects of antibiotics dosage in patients with renal impairment, and the objectives were to evaluate the impact of dose appropriateness on the clinical outcome of antibiotic therapy in renal impairment.

\section{METHODS}

A prospective observational study was carried out in the department of the nephrology, Kasturba Hospital (KH). Data were collected for seven months (September 2016 to April 2017) after the approval of the Institutional Ethics Committee, Kasturba Hospital, Manipal (IEC no - 537/201). Patients (admitted with $\mathrm{AKI}$ and CKD, prescribed with antibiotics, were included in the study. Vulnerable patients, such as pregnant, pediatric, and psychiatric patients were excluded. A predesigned form was used to obtain demographic, biochemical, prescription, and clinical outcomes data. Prior Informed consent was obtained. The baseline infectious parameters were measured at the beginning of antibiotic therapy. . $\mathrm{CrCl}$ was estimated using the Cockroft-Gault method ${ }^{14}$ (conventional method) and the Jelliffe method ${ }^{15}$. The Cockroft-gault equation was used for patients with CKD and AKI, whereas the Jelliffe equation was used in patients with only AKI. Appropriateness of the dose was assessed by referring to standard databases like Micromedex, FDA labels, and product information leaflets published by respective manufacturers. Based on specified outcome measures such as an increase or decrease in WBC, ESR, body temperature, sterile culture, outcomes were termed as significant, followed by which impact of dose appropriateness on clinical outcome was assessed.

Statistical Analysis : Demographic data was expressed in frequency and percentage (\%) followed by mean \pm SD for continuous outcomes. Pearson's Chi-square test was done to identify the association of dose appropriateness and clinical outcome. The statistical analysis was carried out in SPSS version 20.0 (IBM SPSS Statistics for Windows, Version 20.0. Armonk, NY: IBM Corp. Released 2012). A p-value of less than 0.05 was considered significant.

\section{RESULTS}

We identified 163 patients of which 139 fulfilled the inclusion criteria. The reasons for dropouts were missing data or complicated medication regimen. The mean age of patients was $54.75 \pm 13.36$. The study showed that the majority (112) of the patients were diagnosed with CKD. The sample consisted of significant male adults (108). The mean length of stay (LOS) for patients was noted to be $6 \pm 2.5$ days. The detailed demographic details are presented in Table. 1.

Urinary tract infections (UTI) were seen in most of the patients, followed by systemic, bloodstream infections (BSI) and respiratory infections. Gastrointestinal infections were the least (Fig. 1). Most of the patients received monotherapy, followed by dual therapy during their hospitalization. In majority antibiotics were appropriately dosed (63.3\%). Most had a favorable clinical outcome (Fig. 2).

Cefoperazone-sulbactam followed by ceftriaxone, vancomycin, meropenem, levofloxacin, ceftazidime were the common antibiotics administered (Fig. 3). Of 27 with AKI, only 20 were analyzed as they had data on required parametersIn patients with AKI having highly unstable serum creatinine, the Cockroft-Gault method overestimated the $\mathrm{CrCl}$ value with a mean difference of $4.55 \mathrm{ml} / \mathrm{min}$. The relationship between dose appropriateness and clinical outcome in AKI demonstrated that dosing adjustment was not a significant factor $(\mathrm{p}=0.4)$ in achieving a favorable clinical outcome.

In CKD patients, a significant correlation between dose adjustment and clinical outcome $(\mathrm{p}=0.001)$ was obtained (Table. 2). The appropriateness of dosing in CKD patients receiving monotherapy, dual therapy, or triple therapy was compared, which identified that suitable dosing in dual therapy $(\mathrm{p}=0.001)$ had a significant effect on positive clinical outcomes (Table. 3 ). 


\section{DISCUSSION}

Sub optimal dosing of antibiotics has been associated with less than appropriate clinical outcomes and increased resistance. With a smaller number of drugs in the pipeline, careful consideration is required to provide appropriate dosing to improve clinical efficacy and safety ${ }^{16}$. The current study was designed to identify the dose optimization of antibacterial in CKD patients. A similar study conducted by Udani et al., which aimed to follow the dosing guidelines in critically ill patients with renal impairment, presented a mean age of 58 years, which was more than our study population. Further, $58 \%$ of the study population were males as compared to $83 \%$ in ours ${ }^{17}$. Kerr et al. estimated mean number of hospitalization days for CKD was 6.78 days as compared to $6 \pm 2$ days in our study and $6 \pm 3$ in $\mathrm{AKI}^{18}$. Studies specific to antimicrobial dosing in renal impairment were minimal. However, a study by Prajapathi et al. investigated dose appropriateness of all drugs in CKD. The findings showed that $18.89 \%$ of drugs were dosed appropriately, and $81.11 \%$ were not within the dose limit. Similarly, in our study, $63.3 \%$ of the drugs were appropriately dosed, whereas $36.7 \%$ of the drugs did not meet the criteria. The drug which was most inappropriately dosed was levofloxacin in the previously mentioned study whereas in our study, cefoperazone sulbactam was frequently prescribed ${ }^{19}$. Kumar et al. evaluaed antibiotic dosing in a tertiary care hospital and found piperacillin-tazobactam to be most widely used antibiotic in renal impairment followed by meropenem, amoxicillin potassium clavulanate, ciprofloxacin, and ceftriaxone. In our study, the most commonly used antibiotic was cefoperazone-sulbactam followed by ceftriaxone, vancomycin, meropenem, and levofloxacin ${ }^{20}$. The difference observed possibly could be attributed to difference in local sensitivity pattern and institutional guidelines on antibiotic use. Even though, the dosing was assessed to be inappropriate or different compared to recommendations in patients with lower $\mathrm{CrCl}$, around $47 \%$ of patients attained a favorable clinical outcome.85\% of such patients were diagnosed with CKD. This trend shows that there is a divergence in terms of required doses compared to standard recommendations. This poses a great challenge in the identification of predictors for inappropriate dosing of antibiotics for its effective optimisation ${ }^{21,22}$. Frazee et al. and colleagues measured GFR in critically ill patients by six different methods and the estimates were significantly $(\mathrm{p}<0.001)$ different among the methods. The GFR measured was often overestimated, with patients being at higher risk of inadequate dosing $^{23}$. In our study, we found that $\mathrm{CrCl}$ estimated by Cockroft Gault overestimates the value in comparison with the Jelliffe method.

The current study had its limitations. Measurement of $\mathrm{CrCl}$ by collecting urine samples and measuring the creatinine clearance would enable better validation of the methods of $\mathrm{CrCl}$ estimation. In $\mathrm{CKD}$, plasma concentration of antibiotics at different time intervals would help us predicting inaccuracies of dosage regimen if any.

The development of electronic calculators or convenient applications for $\mathrm{CrCl}$ estimation should be considered. Newer methods should be implemented or studied for the estimation of $\mathrm{CrCl}$, specifically in AKI for better dosing optimization and improved clinical outcome.

\section{CONCLUSION}

Antibiotic dosing was not appropriate in $36.1 \%$ of patients but did not influence outcomes in a major way. There was a difference of $4.5 \mathrm{ml} / \mathrm{min}$ in GFR estimation between Jellife and CG formulae and it may be better to follow the former for drug dosing. In CKD, dose appropriateness was found to be a significant factor in achieving a favorable clinical outcome. It is imperative to determine the precise $\mathrm{CrCl}$ and dose accordingly, for the effective management of infections in CKD.

\section{Acknowledgements}

The authors acknowledge Prasanna Kumar Shetty, Department of Pharmacy Practice for his significant contribution in EGFR calculation using various formulae.

\section{Conflict of Interest Statement}

The authors declare no conflict of interest. 


\section{REFERENCES}

1. Askenazi D, Selewski D, Willig L, Warady BA. Acute kidney injury and chronic kidney disease. In: Avery's Diseases of the Newborn.Elsevier; 2018:1280-1300. e1285.

2. Roger P-M, Montera E, Lesselingue D, et al. Risk factors for unnecessary antibiotic therapy: a major role for clinical management. Clin Infect Dis. 2019;69(3):466-472.

3. Corsonello A, Roller-Wirnsberger R, Di Rosa M, et al. Estimated glomerular filtration rate and functional status among older people: A systematic review. Eur J Intern Med. 2018;56:39-48.

4. Heringa M, Floor-Schreudering A, De Smet PAGM, Bouvy ML. Clinical decision support and optional point of care testing of renal function for safe use of antibiotics in elderly patients: a retrospective study in community pharmacy practice. Drugs Aging. 2017;34(11):851-858.

5. Peters L, Olson L, Khu DTK, et al. Multiple antibiotic resistance as a risk factor for mortality and prolonged hospital stay: A cohort study among neonatal intensive care patients with hospital-acquired infections caused by gram-negative bacteria in Vietnam. PloS One.2019;14(5):e0215666.

6. Polasek TM, Kirkpatrick CMJ, Rostami-Hodjegan A. Precision dosing to avoid adverse drug reactions. Ther Adv Drug Saf.2019;10:2042098619894147.

7. Aloy B, Launay-Vacher V, Bleibtreu A, et al. Antibiotics and chronic kidney disease: Dose adjustment update for infectious disease clinical practice. Med Mal Infect. 2020;50(4):323-331.

8. Cox JA, Vlieghe E, Mendelson M, et al. Antibiotic stewardship in low- and middle-income countries: the same but different? Clin Microbiol Infect. 2017;23(11):812-818.

9. Farrington K, Covic A, Nistor I, et al. Clinical Practice Guideline on management of older patients with chronic kidney disease stage $3 \mathrm{~b}$ or higher (eGFR\&lt; $45 \mathrm{~mL} / \mathrm{min} / 1.73 \mathrm{~m} 2$ ): a summary document from the European Renal Best Practice Group. Nephrol Dial Transplant.2017;32(1):9-16.

10. Mulay AV, Gokhale SM. Comparison of serum creatinine-based estimating equations with gates protocol for predicting glomerular filtration rate in indian population. Indian J Nephrol.2017;27(2):124-128.

11. Pelletier K, Lafrance J-P, Roy L, et al. Estimating glomerular filtration rate in patients with acute kidney injury: a prospective multicenter study of diagnostic accuracy. Nephrol Dial Transplant.. 2019.

12. Bouchard J, Macedo E, Soroko S, et al. Comparison of methods for estimating glomerular filtration rate in critically ill patients with acute kidney injury. Nephrol Dial Transplant. 2010;25(1):102-107.

13. Chawla LS, Bellomo R, Bihorac A, et al. Acute kidney disease and renal recovery: consensus report of the Acute Disease Quality Initiative (ADQI) 16 Workgroup. Nat Rev Nephrol. 2017;13(4):241-257.

14. Cockcroft DW, Gault MH. Prediction of creatinine clearance from serum creatinine. Nephron. 1976;16(1):31-41.

15. Jelliffe R. Estimation of creatinine clearance in patients with unstable renal function, without a urine specimen. Am J Nephrol.2002;22(4):320-324.

16. Gill CM, Nicolau DP. Pharmacologic optimization of antibiotics for Gram-negative infections. Curr Opin Infect Dis.2019;32(6):647-655.

18. Kerr M, Bray B, Medcalf J, O'Donoghue DJ, Matthews B. Estimating the financial cost of chronic kidney disease to the NHS in England.Nephrol Dial Transplant. 2012;27(suppl_3):iii73-iii80.

19. Prajapati A, Ganguly B. Appropriateness of drug dose and frequency in patients with renal dysfunction in a tertiary care hospital: A cross-sectional study. J Pharm Bioallied Sci. 2013;5(2):136-140.

20. Kumar A, Khrime D, Bansal N, Pandey A, Varma A. Evaluation of Antibiotic Dose Adjustment in Patients with Renal Insufficiency in a Tertiary Care Center. IJCMR. 2016;3(5):1383-1385. 
21. Shailaja K, Shaji J, George KA, Jacob T, Ubaidulla U. A Prospective Study on Assessment of Therapeutic Management and Clinical Outcomes in Renal Impaired Patients. J Pharm Sci \& Res.2019;11(9):31533159 .

22. Tesfaye WH, Castelino RL, Wimmer BC, Zaidi STR. Inappropriate prescribing in chronic kidney disease: A systematic review of prevalence, associated clinical outcomes and impact of interventions. Int J Clin Pract. 2017;71(7):e12960.

23. Frazee EN, Personett HA, Wood-Wentz CM, Herasevich V, Lieske JC, Kashani KB. Overestimation of glomerular filtration rate among critically ill adults with hospital-acquired oligoanuric acute kidney injury. Journal of Pharmacy Practice. 2016;29(2):125-131.

Table 1: Demographic details of the patients

Table 2: Dose appropriateness vs. clinical outcome in AKI and CKD patients

Table 3: Dose appropriateness vs. clinical outcome in mono, dual and triple therapy

Figure 1. Types of infections

Figure 2: Clinical outcome in patients after treatment

Figure 3: Pattern of antibiotic usage

Hosted file

JECP_Tables.docx available at https://authorea.com/users/335571/articles/461437-doseoptimization-of-antibiotics-in-renally-impaired-patients-in-indian-settings-a-

prospective-observational-study

\section{Hosted file}

JECP_Figures.docx available at https://authorea.com/users/335571/articles/461437dose-optimization-of-antibiotics-in-renally-impaired-patients-in-indian-settings-aprospective-observational-study 